\title{
Allocation Problems, by the Method of Alternative Representation of the Inverse Set, for Values of Cooperative TU Games
}

\author{
Irinel Dragan
}

\begin{abstract}
In earlier works, we introduced the Inverse Problem, relative to the Shapley Value, as follows: for a given n-dimensional vector $L$, find out the transferable utilities' games $(N, v)$, such that $S H(N, v)=L$. The same problem has been discussed further for Semivalues. A connected problem has been considered more recently: find out TU-games for which the Shapley Value equals $L$, and this value is coalitional rational, that is belongs to the Core of the game $(N, v)$. Then, the same problem was discussed for other two linear values: the Egalitarian Allocation and the Egalitarian Nonseparable Contribution, even though these are not Semivalues. To solve such problems, we tried to find a solution in the family of so called Almost Null Games of the Inverse Set, relative to the Shapley Value, by imposing to games in the family, the coalitional rationality conditions.

In the present paper, we use the same idea, but a new tool, an Alternative Representation of Semivalues. To get such a representation, the definition of the Binomial Semivalues due to A. Puente was extended to all Semivalues. Then, we looked for a coalitional rational solution in the Family of Almost Null games of the Inverse Set, relative to the Shapley Value.

In each case, such games depend on a unique parameter, so that the coalitional rationality will be expressed by a simple inequality, determined by a number, the coalitional rationality threshold. The relationships between the three numbers corresponding to the above three efficient values have been found. Some numerical examples of the method are given.
\end{abstract}

Index Terms - Cooperative Transferable Utilities Games, Semivalues, Inverse Problem, Almost Null Family, Coalitional Rationality Thresholds.

\section{INTRODUCTION}

A cooperative transferable utilities game, (TU game), is a pair $(N, v)$, where $N$ is a finite set, the set of players, and $v: P(N) \rightarrow R$, is a characteristic function defined on the Power set of $N$ denoted $P(N)$, with $v(\varnothing)=0$. A model studied in [1], associated to a transportation network, clarifies this concept, namely the fact that for each $S \subseteq N$, a coalition of players, the number $v(S)$, the worth of $v$, will give the win of the coalition, in case that the coalition has been formed. A central problem in Game Theory is the problem of allocating fairly the win of the grand coalition, assuming that the coalition $N$ was formed.

Submitted on April 07, 2021

Published on April 30, 2021.

Irinel Dragan, University of Texas at Arlington, USA.

(e-mail: dragan@uta.edu)
The most famous solutions for this problem are the Shapley Value and the Banzhaf Value, as well as the Semivalues. The Semivalues are due to P. Dubey, A Neyman and R. J. Weber, who defined axiomatically these values. In case of TU-game they may be defined by a vector $p_{n}=\left(p_{n}(1), p_{n}(2), \ldots, p_{n}(n)\right)$, satisfying the normalization condition $\quad \sum_{s=1}^{n} C_{n-1}^{s-1} p_{n}(s)=1, \quad$ where $\quad s=|S|, \quad$ and the components will be weighting the marginal contributions:

$$
S E_{i}(N, v)=\sum_{S: i \in S} p_{n}(s)[v(S)-v(S-\{i\})], \forall i \in N
$$

The Shapley Value and the Banzhaf Value are defined by means of the weighting vectors:

$$
p_{n}(s)=\frac{(s-1) !(n-s) !}{n !}, \forall S \in N
$$

and

$$
p_{n}(s)=\frac{1}{2^{n-1}}
$$

respectively, that may be shown to satisfy the above normalization condition. In [2], A. Puente introduced the class of Binomial Semivalues, by imposing to the weight vector the conditions:

$\lambda_{1}=p_{n}(1), \frac{p_{n}(2)}{p_{n}(1)}=\frac{p_{n}(3)}{p_{n}(2)}=\ldots=\frac{p_{n}(n)}{p_{n}(n-1)}=k$

where $k$ is a constant. Notice that the Shapley Value is not a Binomial Semivalue, while the Banzhaf Value is a Binomial Semivalue, where $k=\frac{1}{2}$. In [3] and [5], we let the ratios from (3) to be variables, that is we take:

$$
p_{n}(1)=\lambda_{1}, p_{n}(2)=\lambda_{1} \lambda_{2}, \ldots, p_{n}(n)=\lambda_{1} \lambda_{2} \ldots \lambda_{n}
$$

The variables are satisfying the normalization condition $\sum_{s=1}^{n} C_{n-1}^{s-1} \lambda_{1} \ldots \lambda_{s}=1$. For example, from (1) and (2), we obtain for the Shapley Value the alternative representation:

$$
S H_{i}(N, v)=\sum_{S: i \in S} \lambda_{1} \lambda_{2} \ldots \lambda_{S}[v(S)-v(S-\{i\}], \forall i \in N
$$


Notice that by using (4) we obtain an alternative representation of any Semivalue, efficient or non-efficient. We shall be interested to give such a representation for the solution of the Inverse Problem, relative to the Shapley Value, which is an efficient value. From this new representation will be derived the main results of the present paper.

In [4], we introduced the Inverse Problem relative to the Shapley Value: for a given nonnegative vector $L$ find out all TU-games $(N, v)$ such that we have $S H(N, v)=L$.

This is a vector space, called the Inverse Set, relative to Shapley Value. Therefore, this Inverse Set may be defined by means of a particular basis, called a Potential Basis, because the coefficients of the basic vectors are the potentials of subgames of the given game. The basis is given by

$W=\left\{W_{T}: T \subseteq N\right\}$,

where

$W_{T}(S)=\sum_{h=0}^{s-t}(-1)^{h} C_{s-t}^{h} \frac{1}{p_{t+h}(t+h)}, \forall S \supseteq T$

and $W_{T}(S)=0$ for all other $S$ Notice that for $S=T$ we have $W_{T}(T)=\frac{1}{p_{t}(t)}, \forall T \subseteq N$.

It was easily seen in [4], that we get an explicit representation of the Inverse Set, by using the potential basis, defined in (6). This representation is given by the formula:

$w=\sum_{S: S \mid \leq n-2} c_{S} W_{S}+a\left(W_{N}+\sum_{j \in N} W_{N-\{j\}}\right)-\sum_{j \in N} L_{j} W_{N-\{j\}}$,

where, beside the arbitrary constants, occur the components of the Shapley Value.

In the Inverse Set, relative to the Shapley Value, it is easy to find games for which the Shapley Value is coalitional rational, that is they belong to the Core of the game. Further, in [3], we introduced and solved a connected problem: for some given vector, find out a game in the Inverse Set, relative to the Shapley Value, where this is unchanged, but it is also coalitional rational. The main idea was to look for such a game in the family of Almost Null Games of Inverse Set, that is the set obtained from (7) as follows:

$$
w=a\left(W_{N}+\sum_{j \in N} W_{N-\{j\}}\right)-\sum_{j \in N} L_{j} W_{N-\{j\}}
$$

From (6), we have the non-zero components of the basic vectors:

$$
\begin{aligned}
& W_{N}(N)=\frac{1}{p_{n}(n)} \\
& W_{N-\{i\}}(N)=\frac{1}{p_{n-1}(n-1)}-\frac{1}{p_{n}(n)}, W_{N-\{i\}}(N-\{i\})=\frac{1}{p_{n-1}(n-1)}
\end{aligned}
$$

Therefore, from (8) and (9) we have the characteristic function of the TU-games in the Almost Null Family of games given by:

$$
w(N-\{i\})=\frac{a-L_{i}}{p_{n-1}(n-1)}, \forall i \in N
$$

$$
w(N)=a\left(\frac{n}{p_{n-1}(n-1)}-\frac{n-1}{p_{n}(n)}\right)-\frac{1}{p_{n}(n)} \sum_{j \in N} L_{j}
$$

where all worth of coalitions of smaller sizes equal zero. In the second section, we get the Alternative Representation for the Family of the Almost Null TU-games from the Inverse Set; the third section is devoted to the problem of finding a TU-game in which the Shapley Value is coalitional rational, and the similar problem for other two values: The Egalitarian Allocation and the Egalitarian Nonseparable Contribution. All the computations will be done by using the Alternative Representation of the Almost Null Family in the Inverse Set relative to the Shapley Value. The last section is discussing new opportunities to consider similar problems to be solved. Some numerical examples are illustrating the procedures used in the proofs.

\section{THE Alternative REPRESENTATION OF THE FAMILY OF ALMOST NULl GAMES}

As we have seen above, we obtained the games (10) found in the Family of Almost Null Games, relative to the Shapley Value, expressed in terms of the weights for this value.

Notice that to obtain the Alternative Representation, we should replace in (10) these weights in terms of the new weights introduced by our formula (4). Remember also the connection between the initial weights for the games with $n$ players and the subgames with n-1 players, that we called the inverse Pascal triangle; this will be first used below in deriving from (10) the new expressions for an Alternative Representation of the Family of the Almost Null Games in the Inverse Set. The main weight appearing in (10) is:

$$
\begin{aligned}
& p_{n-1}(n-1)=p_{n}(n-1)+p_{n}(n)=\frac{p_{n}(n)}{\lambda_{n}}+p_{n}(n)=\frac{1+\lambda_{n}}{\lambda_{n}} p_{n}(n)= \\
& =\lambda_{1} \ldots \lambda_{n-1}\left(1+\lambda_{n}\right)
\end{aligned}
$$

Then, we shall compute the parenthesis from the second formula (10), in which we use the same strategy as above, that is the inverse Pascal triangle and formula (4), to obtain:

$$
\begin{aligned}
& \frac{n}{p_{n-1}(n-1)}-\frac{n-1}{p_{n}(n)}=\frac{n}{\lambda_{1} \ldots \lambda_{n-1}\left(1+\lambda_{n}\right)}-\frac{(n-1)}{\lambda_{1} \ldots \lambda_{n-1} \lambda_{n}}= \\
& =\frac{1+\lambda_{n}-n}{\lambda_{1} \ldots \lambda_{n}\left(1+\lambda_{n}\right)}
\end{aligned}
$$

From (10), (11) and (12), we get the Alternative Representation for the Family of Almost Null Games in the Inverse Set, relative to the Shapley Value: 
$w(N-\{i\})=\frac{a-L_{i}}{\lambda_{1} \ldots \lambda_{n-1}\left(1+\lambda_{n}\right)}, \forall i \in N$

$w(N)=\frac{\left(1+\lambda_{n}-n\right) a+\sum_{j \in N} L_{j}}{\lambda_{1} \ldots \lambda_{n}\left(1+\lambda_{n}\right)}$

$w(S)=0, \forall S,|S| \leq n-2$

(see also [5]).

\section{COALITIONAL RATIONALITY AND THE INVERSE PROBLEM}

We consider the problem: a nonnegative n-vector $L$ being given, find out a TU-game such that the Shapley Value equals $L$, and this vector is coalitional rational, that is it belongs to the Core. In this section, beside this problem, we shall discuss the similar problems for two other efficient values: The Egalitarian Allocation and the Egalitarian Nonseparable Contribution. Recall that the following conditions define the Core:

$$
\begin{aligned}
& \sum_{j \in N} x_{j}=w(N) \\
& \sum_{j \in S} x_{j} \geq w(S), \forall S \subset N
\end{aligned}
$$

for an n-vector $x$ and this vector is considered a fair sharing of the worth of N. As we have the efficiency holding for all three values, we should impose only the group of the second conditions (14).

\section{A. The Shapley Value}

Above, we can find, from (2) and (4), that we have:

$\lambda_{n}=n-1$

$\lambda_{1} \lambda_{2} \ldots \lambda_{n-1}\left(1+\lambda_{n}\right)=\frac{1}{n-1}$

so that the formulas (13) become:

$$
\begin{aligned}
& w(N-\{i\})=(n-1)\left(a-L_{i}\right), \forall i \in N \\
& w(N)=\sum_{j \in N} L_{j} \\
& w(S)=0,|S| \leq n-2
\end{aligned}
$$

This is the general expression for the games in the Family of Almost Null Games from the Inverse Set relative to the Shapley Value. Hence, we get the result:

Theorem 1: A non-negative efficient vector $x \in R^{n}$ which satisfies the inequalities:

$$
\sum_{j \in N-\{i\}} x_{j} \geq(n-1)\left(a-L_{i}\right), \forall i \in N
$$

will be a solution of the inverse problem with coalitional rationality, for a game in the Family of Almost Null Games of the Inverse Set, relative to the Shapley Value.

As a consequence of Theorem 1, the set of solutions for our problem stated above is the infinite set defined by (16), where the unique parameter satisfies the condition:

$a \leq \frac{1}{n-1}\left[w(N)+(n-2) M I N_{i} L_{i}\right]=\alpha$

Example 1: Consider the 3-person game:

$v(1)=v(2)=v(3)=0, v(1,2)=22, v(1,3)=v(2,3)=$
$=18, v(1,2,3)=25$

Compute the Shapley Value; we have:

$\operatorname{SH}(N, v)=(9,9,7)$

and notice that this is not coalitional rational. Compute $\alpha=16$, and use the formula (16) to get one solution, when we take this maximal value. We obtain the game:

$$
\begin{aligned}
& w(1)=w(2)=w(3)=0, w(1,2)=18, w(1,3)=w(2,3)= \\
& =14, w(1,2,3)=25
\end{aligned}
$$

which gives the same Shapley Value, but now it is coalitional rational. Whatever other value of the parameter in the interval $[0,16]$ would give a solution.

\section{B. The Egalitarian Allocation}

The value called the Egalitarian Allocation is given by:

$E A_{i}(N, v)=\frac{v(N)}{n}, \forall i \in N$

and it is an efficient value. We consider the similar problem given a vector $M \in R^{n}$, find out a game $(N, w)$, such that $E A(N, w)=M$, and this is coalitional rational.

Now, we do not have an Inverse Set, relative to the Egalitarian Allocation, so that we shall be using a new idea: find out a solution in the Inverse Set, relative to the Shapley Value. As the Shapley Value is efficient, and the Egalitarian Allocation is also efficient, to solve the new problem we should impose only the coalitional rationality condition.

As above, we shall try to find a solution in the Family of Almost Null Games of this set, for which we have the general expression (16). Notice that in the right hand sides will occur the Shapley Value of the same game. The coalitional rationality condition is:

$(n-1) \frac{w(N)}{n} \geq(n-1)\left(a-L_{i}\right), \forall i \in N$

or, after simplifying, we get:

$a \leq \frac{w(N)}{n}+\operatorname{MIN}_{i} L_{i}=\beta$ 
Theorem 2: A non-negative efficient vector $x \in R^{n}$ is a solution of the inverse problem with coalitional rationality for the Egalitarian Allocation, if the parameter satisfies the inequality (24). A solution will be obtained by choosing in (16) a parameter value $a \in[0, \beta]$, (see [6]).

Recall that we got in both cases, some semi-infinite interval determined by a number called the threshold of coalitional rationality. It is interesting to find out the inequality between these thresholds. We have:

$$
\begin{aligned}
& \alpha-\beta=\frac{1}{n-1}\left[w(N)+(n-2) M I N_{i} L_{i}\right]-\left[\frac{w(N)}{n}+\operatorname{MIN}_{i} L_{i}\right]= \\
& =\frac{1}{n(n-1)} w(N)-\frac{1}{n-1} \operatorname{MIN}_{i} L_{i}=\frac{1}{n(n-1)}\left[w(N)-n M I N_{i} L_{i}\right] \geq 0
\end{aligned}
$$

which, together with theorem 2 , proves the result:

Corollary 3: The Shapley Value and the Egalitarian Allocation have both an inverse solution with coalitional rationality, if the parameter satisfies $a \in[0, \beta]$.

Example 2: Consider the same 3-person game as in example 1 and compute the Egalitarian Allocation: $E A(N, w)=\left(\frac{25}{3}, \frac{25}{3}, \frac{25}{3}\right)$, which is efficient but not a coalitional rational vector. Compute the common threshold for coalitional rationality $\beta=\frac{46}{3}$, and find out a solution by choosing for the parameter the maximal value in the interval. From (16) we get:

$w(1)=w(2)=w(3)=0, w(1,2)=\frac{50}{3}, w(1,3)=w(2,3)=$

$=\frac{38}{3}, w(1,2,3)=25$

We can check easily that the Shapley Value and the Egalitarian Allocation of this game are unchanged, and they are also efficient and coalitional rational.

\section{The Egalitarian Nonseparable Contribution}

The value called above the Egalitarian Nonseparable Contribution, due to T. Driessen and Y. Funaki (see [7]), is given by:

$$
\begin{aligned}
& \operatorname{ENSC}_{i}(N, v)=v(N)-v(N-\{i\})+\frac{1}{n}\{v(N)- \\
& \left.-\sum_{j \in N}[v(N)-v(N-\{j\})]\right\}, \forall i \in N .
\end{aligned}
$$

We shall be looking for a solution with coalitional rationality, like in 3.2, in the Family of Almost Null Games of the Inverse Set, relative to the Shapley Value. However, this time we shall prove first that for all games in this family the Egalitarian Nonseparable Contribution is unchanged. So, compute the terms from formula (28) by using the representation for the games in the Family of Almost Null Games shown in (16):

$$
\frac{1}{n}\left\{w(N)-\sum_{i \in N}[w(N)-w(N-\{i\}]\}=(n-1) a-\frac{2(n-1)}{n} w(N)\right.
$$

From (16) and (29), we obtain the expression of Egalitarian Nonseparable Contribution

$$
\operatorname{ENSC}_{i}(N, w)=(n-1) L_{i}-\frac{n-2}{n} w(N), \forall i \in N
$$

Notice the very important fact that the formula (30) does not depend on any parameter, which means that the value is unchanged relative to the original values on the same games. Now, impose the coalitional rationality condition; starting from (16), we have:

$$
w(N)-\operatorname{ENSC}_{i}(N, w) \geq(n-1)\left(a-L_{i}\right), \forall i \in N
$$

or by using the expression of Egalitarian Nonseparable Contribution (30), we obtain:

$a \leq \frac{2}{n} w(N)=\gamma$

Theorem 4: A non-negative efficient vector $x \in R^{n}$ is a solution of the inverse problem with coalitional rationality for the Egalitarian Nonseparable Contribution if the parameter satisfies the inequality (32). A solution will be obtained by choosing in (16) a parameter value $a \in[0, \gamma]$, (see [8]).

Recall that we computed the coalitional thresholds for the two values discussed above and the smaller is the threshold for the Egalitarian Allocation. Let us see the inequality connecting this smaller threshold to the new threshold for the Egalitarian Nonseparable Contribution. From (32) and (24), we have:

$$
\gamma-\beta=\frac{1}{n}\left[w(N)-n M I N_{i} L_{i}\right] \geq 0
$$

From (33) follows the result:

Corollary 5: The Shapley Value, the Egalitarian Allocation and the Egalitarian Nonseparable Contribution have all three a solution with coalitional rationality, if the parameter value will satisfy $a \in[0, \beta]$. As shown in [8], we have also $\gamma \geq \alpha \geq \beta$.

Example 3: Consider the same game like in examples 1 and 2; the threshold of coalitional rationality, given by (32), is $\gamma=\frac{50}{3}$, satisfying the condition of Theorem 4 . In this way, to get a solution with coalitional rationality for all three values, we should choose $a \in[0, \beta]$. In (26) we have the game obtained for the maximal value of the parameter in this interval.

Compute the Egalitarian Nonseparable Contribution for this game. The contributions of the players to the grand coalition are: 


$$
w(N)-w(2,3)=w(N)-w(1,3)=\frac{37}{3}, w(N)-w(1,2)=\frac{25}{3}
$$

the average of leftover is:

$$
\frac{1}{3}\left\{w(N)-\sum_{j \in N}[w(N)-w(N-\{i\})]\right\}=-\frac{8}{3}
$$

and the Egalitarian Nonseparable Contribution is the vector:

$$
\operatorname{ENSC}(N, w)=\left(\frac{29}{3}, \frac{29}{3}, \frac{17}{3}\right)
$$

that is the same as the value computed for the initial game. Of course, the same will be true for the other two values. Now, we can check that in the new game (26) they are all coalitional rational. A similar situation will occur if we choose another value for the parameter, in the same interval $[0, \beta]$.

\section{DISCUSSIONS AND REMARKS}

In the present paper, we solved a problem connected to the inverse problem relative to the Shapley Value, by using the Family of Almost Null Games of the Inverse Set. Earlier, we discussed the problem for the Shapley Value. Obviously, we may find in the Inverse Set, relative to the Shapley Value, some solution which is coalitional rational, but does not belong to the Family of the Almost Null Games. This could be a good topic for some future research, namely: find out all games in the inverse set, which are solutions of our problem. In earlier papers, (see [6] and [8]), we have found solutions in the Inverse Set relative to the Shapley Value, for Egalitarian Allocation and Egalitarian Nonseparable Contribution. Obviously, another research topic may be that of finding games in the Inverse Set, relative to each of these values and look for a coalitional rational solution in a similar Family of Almost Null Games of such inverse sets. Finally, another possible topic may be that of discussing similar problems for other efficient values of TU-games. As noticed in an earlier work, (see [9]), it is possible to discuss the same problem for non-efficient values, but a more general definition of coalitional rationality will be needed.

The main purpose of this paper was to see whether, or not, some results may be obtained by starting from alternative forms of the well known formulas for the values discussed.
[3] Dragan, I., (2014), Coalitional Rationality of the Shapley Value and other efficient values of cooperative TU games, American Journal of Operations Research, vol.4,4, 228-234.

[4] Dragan, I., (1991), The Potential Basis and the weighted Shapley Value, Libertas Mathematica, 11, 133-150.

[5] Dragan, I., and Dehez, P., (2020), Alternative Representation of Semi values, the Inverse Problem and the Coalitional Rationality, Journal of Mathematics and Statistics Sciences, Vol.6,1, 68-84.

[6] Dragan, I., (2018), Egalitarian Allocations and the Inverse Problem for the Shapley Value, American Journal of Operations Research, vol.8,6, 448-456.

[7] Driessen, T., and Funaki, Y., (1997), The Egalitarian Nonpairwise Averaged Contribution for TU Games, In: Parthasarathy T., et al., Eds., Game Theoretical Applications to Economics and Operations Research, Kluwer Academic Publishers, Amsterdam, 51-66.

[8] Dragan, I., (2019), On the Coalitional Rationality and the Egalitarian Nonseparable Contribution, Applied Mathematics, vol.10,5, 363-370.

[9] Dragan, I., (2015), Coalitional Rationality of the Banzhaf Value and other non-efficient values of cooperative TU games, Applied Mathematics, vol.6,12, 2069-2076.

\section{REFERENCES}

[1] Dragan,I., (2015), On the Gas Routing via Game Theory, American Journal of Operations Research, Vol.5,4, 288-292.

[2] Puente,A., (2000), Contributions to the representability of simple games and to the calculus of solutions for this class of games, Ph. D. Thesis, Univ. Catalonia, Barcelona, Spain. 